\title{
An Energy-efficient Wireless Sensor Network for Precision Agriculture
}

\author{
Herman Sahota \\ Ratnesh Kumar \\ Ahmed Kamal \\ Jing Huang \\ Dept. of Electrical and Computer Engineering, Iowa State University, Ames, IA 50011
}

\begin{abstract}
The use of wireless sensor networks is essential to implementation of information and control technologies in application areas such as precision agriculture. We design MAC and Network layers for a wireless sensor network deployed for a precision agriculture application which requires periodic collection of sensor readings from fixed locations in a field. The Physical layer consists of a radio which operates in multiple power levels in the transmit mode and multiple sensitivity levels in the receive mode. The MAC layer is designed to save energy during the wake-up synchronization phase. The network layer is designed to custom fit the needs of the application, namely periodic data collection from fixed locations, and to minimize the energy consumption through balancing the communication load. The design of various protocol layers involves a cross-layer design strategy, taking into consideration the requirements and the characteristics of the application.
\end{abstract}

\section{INTRODUCTION AND RELATED WORK}

Precision agriculture refers to the use of information and control technologies in agriculture [1]. Effective soil data collection and processing is a key requirement in this application to use the resources effectively for maximizing the crop yield and minimizing the impact on the environment. In this paper we describe our work on a sensor network for such a precision agriculture application.

Our MAC and network layers are designed to meet the requirements of the application (periodic collection of sensordata and energy efficiency). Soil monitoring application does not have latency as a strict requirement, but data integrity with a certain confidence has to be guaranteed. Our scheme is based on adjusting the receiver sensitivity level and sender transmission power during the wake-up phase to trade off the energy consumed at the receiver for that at the transmitter of the wake-up signal. Routing strategy further balances the energy consumption across all the nodes in the field.

Energy efficiency during radio communication is achieved by minimizing idle-listening, overemitting (transmission when receiver is not ready), collisions and overhearing (node receiving a packet not destined for itself). Protocols such as such as S-MAC [2] and T-MAC [3] use a periodic listensleep schedule to minimize idle-listening. B-MAC [4] and WiseMAC [5] use preamble sampling scheme to reduce the radio duty cycle. A TDMA based protocol can minimize collisions and overhearing but at a higher overhead cost of near-perfect synchronization.

This work was supported in part by the National Science Foundation under the grants CNS-0626822, NSF-ECS-0601570, NSF-ECCS-0801763, NSFCCF-081141, and NSF-ECCS-0926029.
In the following sections we present the network model, description of our MAC and Network layers, simulation results and conclusions.

\section{NeTwORK MODEL}

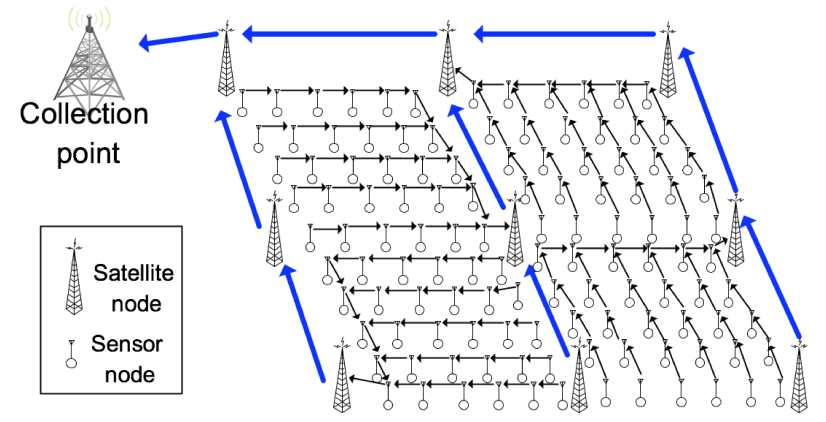

Fig. 1. Wireless sensor network, showing sensor and satellite station deployment.

Our precision agriculture farm is divided into regular square sensor fields (see Figure 1). Within each field, sensors are deployed in a rectilinear grid form, with grid size of $50 \mathrm{~m} \times 50 \mathrm{~m}$, and at a depth of $30 \mathrm{~cm}$. To conserve energy, only adjacent nodes are able to communicate directly with each other. The satellite stations at the corners of each field have a transmission range that can cover all nodes in the field. The satellite stations do not perform any sensing operation, but they serve three purposes:

1) they collect information from the sensor nodes in a field and relay the collected information to a base station,

2) they dispatch routing and scheduling information to the sensors in the field, and

3) they participate in the sensor localization process.

Due to the first point above, the routing is based on a two-level hierarchy (field-level versus satellite-station level), which can simplify routing and also save energy of the sensor nodes. Moreover, the second point also reduces the computationalcomplexity and hence energy consumption of the sensor nodes. The third point above is the subject of our future work.

The routing computes routes over sensor nodes in the field such that the different satellite stations take turns in gathering data, so that energy consumption is balanced. Additionally the routing strategy has the advantage of withstanding failures and achieving a certain level of resilience, without adding any extra measures for this purpose. 
Ours is a hybrid network, combining features from both ad-hoc and infrastructured networks. Also note that the our network model is scalable due to the reason that the energy consumption is a linear function of the number of nodes.

\section{ENERGY EFFICIENT WAKE-UP AND DATA COLLECTION}

Energy efficiency is achieved by reducing the energy consumed by the receiver of the wake-up synchronization signal at the PHY/MAC layer level, and by using routing and scheduling to achieve load balancing and keeping the number of internode communications per round to a minimum. A Round refers to period of time where the sensed data from all the nodes in a field are forwarded to the particular satellite node acting as the sink. The role of the sink is rotated among the satellite nodes of a field with each round (This increases the measure of resilience against node failures). A collection of consequetive rounds in which each satellite node has acted once as a sink is termed as a macro-round.

Due to the large sleep periods, the relative clock drifts between neighboring nodes can be significant. Typical drift in crystals employed in clocks in sensor nodes is of the order of $30 \mathrm{ppm}$. To reduce the energy consumed during the wakeup synchronization phase, the receiver of the wake-up signal listens at a lower sensitivity level while waiting for a wakeup signal from a neighboring node which is transmitted at a higher power level. We term the mode of operation of a node while transmitting the wake-up signal as ping, and the mode of operation of the receiver circuit in the degraded sensitivity as drowsy, see Figure 2. Thus we make a trade-off between the energy consumed at the receiver of the wake-up signal and that of its sender.

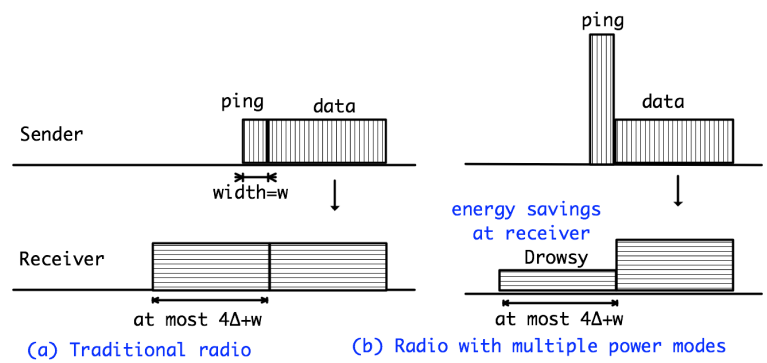

Fig. 2. Wake-up: (a) Existing versus (b) Proposed schemes.

In the worst case of maximum relative clock drift between two nodes, the receiver of a wake-up signal idle-listens for a time that is of the order of $4 \Delta$, where $\Delta$ is the maximum clock drift during the period since last synchronization, in order to make sure it can capture the wake-up synchronization signal from its downstream neighbor. Therefore, the energy consumed by the receiver can be significant if $\Delta$ is large, which is the case for longer sleep periods. The appearance of the term " $4 \Delta$ " can be understood as follows: The sender of the wake-up signal may wake up $\Delta$ time units earlier than scheduled, and in order to not miss the ping, the receiver of the wake-up signal must target waking up $2 \Delta$ units earlier than scheduled (so that it will wake up at the latest by $\Delta$ units prior to sender's scheduled wake-up time). But then the receiver of the wake-up signal may wake up as early as $3 \Delta$ units prior to the sender's scheduled wake-up time whereas the sender may wake up as late as $\Delta$ unit after its own scheduled wake-up time. As as a result the receiver may remain drowsy for at most $4 \Delta$ units before it starts to witness the ping signal. To ensure that $\Delta$ does not grow unbounded, the clocks need to be synchronized periodically, and in our application this is done at the beginning of each round.

The degraded receiver sensitivity can be achieved by simplifying some parts of the receiver-circuitry. For example, we can by-pass some amplifier stages, and save on the energy they consume for their operation. The wake-up signal is a signal of a very short duration and carries no data and its sole purpose is to generate an interrupt in the receiver's circuit. Figure 2 illustrates the energy saving achieved by using the drowsy and ping modes as opposed to the regular transmit and receive levels. Denoting by $P_{t}$ and $P_{r}$ the power consumption during normal transmit and receive, and $P_{p}$ and $P_{d}$ that during ping and drowsy modes, the energy saved during a wake-up synchronization can be up to $(4 \Delta+w)\left(P_{r}-P_{d}\right)-w\left(P_{p}-P_{t}\right)$ (the first term denotes the energy saved at the receiver, and the second term subtracts the extra energy needed for ping). Here, $w$ denotes the duration of the ping pulse.

Such a wake-up strategy has been proposed by the use of RFID technology in [6]: Each sensor node is equipped with a tag and a reader. The reader at one node is used to send the ping to the tag of a neighboring node to awaken the latter. The tag, on receiving the signal, generates an interrupt which activates the antenna system for regular data communication. Here the drowsy mode is realized by drawing energy from the received signal itself.

\section{A. MAC Layer Design}

In our setting, at the beginning of each round the routing, schedule and initial clock reset information is delivered to all the nodes in the field. The nodes put their radios to sleep after receiving this information, while setting their timers to fire at the scheduled time for communication. Due to clock drifts, one of the nodes in the communication needs to idle-listen for a period $4 \Delta$ as discussed above. Due to the convergecast nature of the traffic, the receiver of the data is responsible for sending the ping signal to all its senders (rather than the other way). Also in our setting, we allow up to two retransmissions in case of data loss. Figure 3 shows the time-line of events for our MAC protocol for one receiver versus two upstream senders in the scenario, when the first transmissions from both the senders fail, and they are given a second chance to transmit their data. Henceforth, we will refer to our Ping-Drowsy MAC protocol as PD-MAC.

\section{B. Network layer}

Multi-hop routes from all the sensor nodes to a sink are computed for each round. Energy efficiency is achieved by way of load-balancing and avoiding more than one successful 


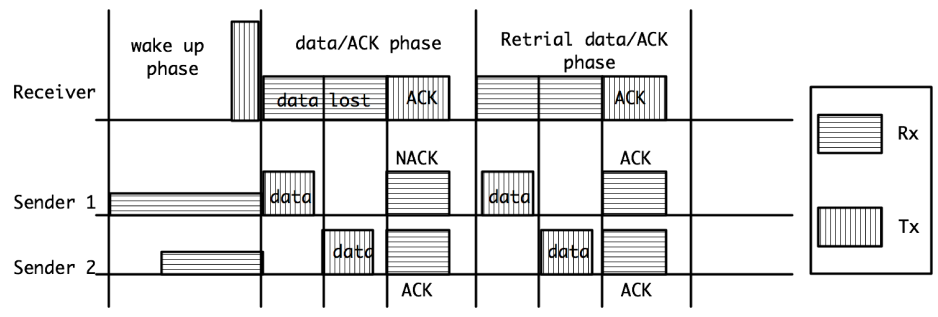

Fig. 3. Sample time-line of events in PD-MAC.

transmission by each node in each round (since each transmission incurs the additional energy for wake-up synchronization). That is, a node must forward to a downstream node only when it has received the data from all of its upstream nodes to avoid multiple transmissions. In each round, the nodes forward their data to a sink located at one of the corners of the field. The role of the sink is rotated among the corners of the field to further balance the energy consumption in the field and to increase the resilience against node failures. Also by appropriate scheduling, interference between sensors in adjacent fields is avoided. Within a round, a node forwards its data to a neighboring node that is closer to a designated sink node. In case of multiple choices for such a neighbor, the data are forwarded to one with maximum remaining energy level. This routing helps balance out the energy consumption across the entire sensor field, as demonstrated by our simulation results.

Further, the routes determine the order in which nodes must forward their data. The scheduling of the nodes must be performed to determine the exact timings of events such as, when a node shall initiate wake-up (by generating ping), when shall a node go into drowsy (to capture a ping), and when shall a node transmit its data together with the data it has collected from its upstream nodes. This schedule is formed to ensure that the data collection takes place with as few pings as possible. For this, all the senders to a receiver are grouped and awakened together by a single ping from the receiver, and further only those senders are allowed retransmission whose communications failed (at most two retransmissions are allowed in the current scheduling algorithm, but this can be adjusted to meet the requirements of the data delivery ratio). The routing and the scheduling information is computed by the corner satellite stations, and is then distributed to the nodes in the field.

\section{Simulation RESUltS AND INITIAL IMPLEMENTATION}

We obtained performance results using the event driven simulation framework of TOSSIM [7] to generate trace files which were analyzed in python. To compare the performance of PDMAC with SMAC (without RTS/CTS since our scheduling ensures no contention), we simulated the behavior of two nodes, a sender and a receiver. The initial listen period of SMAC was chosen to ensure that the two nodes are able to synchronize with each other to follow the same sleep-listen duty cycle, which we choose to be $50 \%$, and the listen period is chosen sufficiently long so as to allow transmission of three data and three corresponding acknowledgements. The sender node sets its timer to wake up once a round to send the collected data to the receiver. Note that in the worst case the nodes are in active mode for one listen period only in addition to the initial active period for synchronization. Clock drift in the sleep timer was modeled using a uniform random number generator over $\left[T_{\text {schedule }}-\Delta, T_{\text {schedule }}+\Delta\right]$.

The current amperage values used for the simulation (along with other parameters) have been taken from the datasheet of CC1110, see Table I. We set the current drawn during the drowsy mode to $10.0 \mathrm{~mA}$ which is roughly half of that in the regular receive mode.

TABLE I

PARAMETER VALUES USED IN SIMULATION.

\begin{tabular}{|l|l|}
\hline Bitrate & $1.2 \mathrm{kbps}$ \\
\hline Transmit current drawn & $15.0 \mathrm{~mA}$ \\
\hline Receive current drawn & $19.8 \mathrm{~mA}$ \\
\hline $\begin{array}{l}\text { Drowsy current drawn } \\
\text { (PDMAC only) }\end{array}$ & $10.0 \mathrm{~mA}$ \\
\hline $\begin{array}{l}\text { Ping current drawn (PDMAC } \\
\text { only) }\end{array}$ & $33.5 \mathrm{~mA}$ \\
\hline Data packet & 8 bytes \\
\hline Ack packet & 4 bytes \\
\hline Sync packet (SMAC only) & 12 bytes \\
\hline $\begin{array}{l}\text { Ping pulse duration (PDMAC } \\
\text { only) }\end{array}$ & $1 \mathrm{~ms}$ \\
\hline $\begin{array}{l}\text { SMAC initial listen period be- } \\
\text { fore transmiting sleep schedule }\end{array}$ & $2 \Delta+T_{\text {synchpacket }}$ \\
\hline SMAC listen period & $3\left(T_{\text {data }}+T_{\text {ack }}\right)$ \\
\hline SMAC sleep period & $3\left(T_{\text {data }}+T_{a c k}\right)$ \\
\hline Clock frequency & $33 \mathrm{kHz}$ \\
\hline PDMAC max drowsy duration & $4 \Delta+T_{\text {ping }}$ \\
\hline Packet error rate & 0.2 \\
\hline
\end{tabular}

The result of energy consumption comparison between our MAC and SMAC is shown in Figure 4 from which we see that our MAC protocol achieves a much better performance than SMAC. This is due to the fact that in SMAC, there is more overhead in the wake-up synchronization phase because the nodes listen at the normal receive power level. In addition, they need to exchange clock synchronization information to synchronize their local clocks with each other.

Next, we simulated the behaviour of the sensors laid out in a square field. Figure 5 shows the energy consumption for 100 nodes in a $10 \times 10$ field for the cases of a traditional radio versus a radio with multiple-power modes and with and without sink node rotation over 10 macro-rounds. For the case of no-rotation there is a large variation in the energy consumed by the nodes because the nodes that are closer to the sink node (shown as the lower id numbers) get more drained than the others. The most balanced energy consumption among the three cases is observed in the case of the radio with multiple power modes and when load balancing is applied at the routing layer and effectively implemented at the scheduling layer.

Figure 6 shows the difference between the energy consumed by the most energy drained node and that by the least energy drained node when the field size is kept constant at $5 \times 5$, 


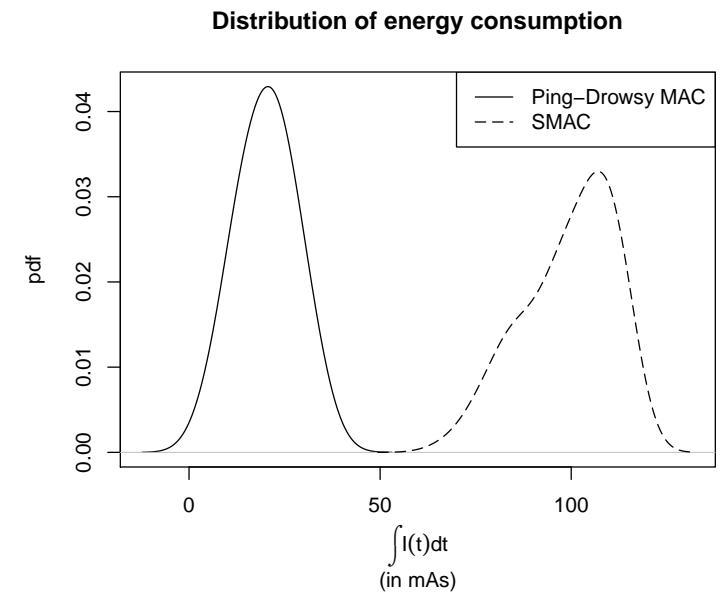

Fig. 4. Energy consumption distribution for SMAC and our MAC protocol.

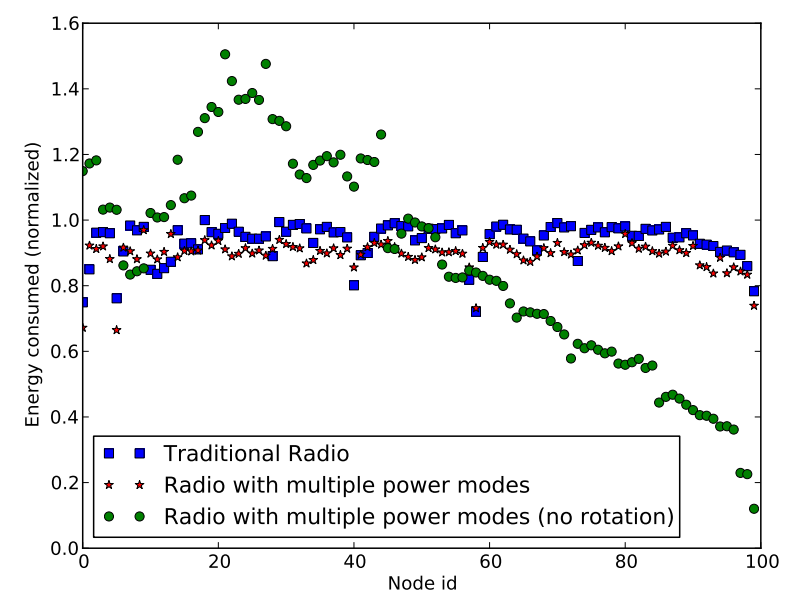

Fig. 5. Energy consumption of 100 nodes over 10 macro-rounds.

but the number of macro-rounds is varied from 1 to 10 . The consistently lower values for the radio with multiple transmit power and receiver sensitivity levels confirm a more balanced energy consumption in the field.

As a proof of concept, we have implemented the protocol for the case of two nodes, i.e., one uplink node and one downlink node. The hardware of the nodes is based on CC1110 Systemon-Chip with low-power RF transceiver and $8051 \mathrm{MCU}$ from Texas Instruments. Specifically, each node is composed of a CC1110 evaluation module plugged into a SmartRF04 evaluation board whose LCD, LEDs and buttons are readily available for monitoring and control. The code is written in C language and compiled under IAR Embedded Workbench. The hardware allows radio transmission in multiple power levels and also allows the user to change the receiver sensitivity.

\section{CONClusion}

We have presented the design of MAC and network layers in a wireless sensor network application for precision agri-

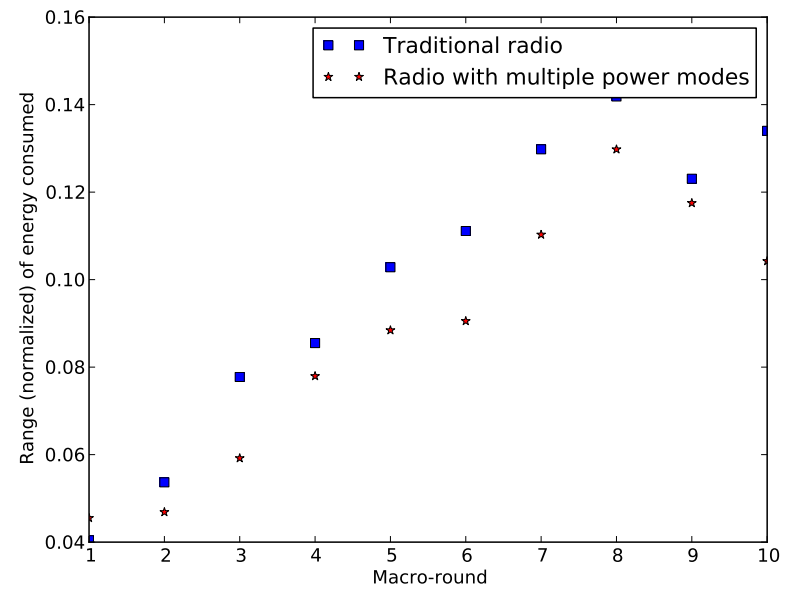

Fig. 6. Difference between the energy consumed by most energy drained node and the least energy drained node versus number of macro-rounds.

culture that requires periodic collection of data from fixed locations, and that has long sleep periods. Due to the large drift in the local clocks of the nodes, considerable energy is consumed during the wake-up synchronization phase of the communication. Our network stack is designed with the goal of energy efficiency during wake-up synchronization by trading off the energy consumed at the receiver for that at the transmitter. Simulation results confirm that PD-MAC is more energy efficient than SMAC for the application under discussion. Further simulations justified the need for a radio with multiple transmit power levels as well as multiple receiver sensitivity levels. Our future work plan includes enhancing the protocol to incorporate fault management (both detection and mitigation). Field tests of the network are underway, whereas we have successfully tested our MAC protocol employing the new wake-up synchronization strategy.

\section{REFERENCES}

[1] J. Burrell, T. Brooke, and R. Beckwith. Vineyard computing: Sensor networks in agricultural production. IEEE Pervasive Computing, 3(1), 2004.

[2] W. Ye, J. Heidemann, and D. Estrin. Medium access control with coordinated adaptive sleeping for wireless sensor networks. IEEE/ACM Trans. Netw., 12(3), 2004.

[3] T. van Dam and K. Langendoen. An adaptive energy-efficient mac protocol for wireless sensor networks. In Proceedings of SenSys '03, NY, USA, 2003. ACM.

[4] J. Polastre, J. Hill, and D. Culler. Versatile low power media access for wireless sensor networks. In Proceedings of SenSys '04, NY, USA, 2004. ACM.

[5] A. El-Hoiydi and J.-D. Decotignie. Wisemac: an ultra low power mac protocol for the downlink of infrastructure wireless sensor networks. In Proceedings of ISCC '04, Washington, DC, USA, 2004.

[6] A. Ruzzelli, R. Jurdak, and G. O'Hare. On the rfid wake-up impulse for multi-hop sensor networks. In ACM SenSys 2007. ACM, November 2007.

[7] P. Levis, N. Lee, M. Welsh, and D. Culler. Tossim: accurate and scalable simulation of entire tinyos applications. In Proceedings of SenSys '03, New York, NY, USA, 2003. 